Pacific Journal of Mathematics

INNER INVARIANT SUBSPACES 


\title{
INNER INVARIANT SUBSPACES
}

\author{
GARY S. ITZKOWITZ
}

We single out a special subclass of the invariant subspaces which we call the inner invariant (i.i.) subspaces. A closed subspace $K$ of a Hilbert space $H$ is said to be $i . i$. for a linear operator $T$ (with domain $D$ ) if: (1) $T(K \cap D) \subseteq K$, (2) $\{T(K \cap D)+(K \cap D)\}^{-}=K$, and (3) $x \in D \backslash K \Rightarrow T x \notin K$. This generalizes subspaces invariant for both $T$ and $T^{-1}$ when the latter exists.

Some of the results in this paper are:

1. Let $\lambda \in \mathbf{C}$. If $|\lambda|<1$ then $K$ is $i$.i. for $U-\lambda$ where $U$ is the shift on Hardy space $H^{p}$ iff $K=g H^{p}$ where $g$ is inner and $g(\lambda) \neq 0$. If $|\lambda| \geqq 1$, then $K$ is $i . i$. for $U-\lambda$ iff $K=g H^{p}$ where $g$ is inner. 2. There is an isometry $J$ from $H^{2}$ onto $L^{2}(0, \infty)$ such that the $i$.i. subspaces of $V+1$ (where $V f(x)=\int_{0}^{x} f(y) d y$ ) are precisely the subspaces $J\left(g H^{2}\right)$ for $g$ an inner function. 3. Any skew-symmetric simple operator with defect indices $(0,1)$ is isomorphic with $V$ and $V^{-1}$.

1. Introduction. In $\S 2$ below we present the definitions of invariance and inner invariance for (not necessarily bounded) operators. Then their fundamental properties are analyzed.

In $\S 3$ we calculate the inner invariant subspaces of the shift operator in several settings. In one setting we describe the inner invariant subspaces of the shift on the Hardy space $H^{p}$. Then we generalize this result.

In $\S 4$ we consider the unbounded Volterra operator $V$. We first characterize this operator abstractly and then use this to get the result that on $L^{2}(0, \infty)$ integration and differentiation (i.e., $V$ and $V^{-1}$ ) are isometrically isomorphic.

Finally, in $\$ 5$, we describe the inner invariant subspace structure of the unbounded operator $V+1$.

2. Definitions and basic properties. We make the following conventions. We work in a Hilbert space $H$ and closed linear subspaces $K$. A linear, though not necessarily bounded, operator on $H$ will be denoted by $T$ with linear domain $D=D(T)$. If $T^{-1}$ exists, we write $D^{-1}$ for $D\left(T^{-1}\right) \equiv T(D(T))$.

If $R$ and $S$ are linear subspaces of $H$ then $R+S$ is the linear subspace generated by the elements of $R$ and $S$. The closure of $R$ in $H$ is denoted by $\bar{R}$ or $\{R\}^{-}$. 
Definition 2.1. A closed subspace $K$ of $H$ is invariant for $T$ if:

(i) $T(K \cap D) \subseteq K$;

(ii) $\{T(K \cap D)+(K \cap D)\}^{-}=K$.

This definition allows for the extreme that neither $T(K \cap D)$ nor $K \cap D$ alone is dense in $K$ yet $K$ is still an invariant subspace for $T$.

Definition 2.2. A closed subspace $K$ is inner invariant for $T$ if it is invariant for $T$ and in addition it satisfies the following property:

(iii) $x \in D \backslash K \Rightarrow T x \notin K$.

The following example shows that an invariant subspace is not necessarily inner invariant and hence that invariance and inner invariance are different.

EXAMPLE 2.3. Consider the shift operator $s$ on $l^{2}$ where $s\left(\left[a_{0}, a_{1}, a_{2}, \cdots\right]\right)=\left[0, a_{0}, a_{1}, a_{2}, \cdots\right]$. Let $K=\left\{\left[a_{n}\right]_{n=0}^{\infty} \mid a_{0}=0\right\}$; then it is a straightforward matter to show that $K$ is an invariant subspace for $s$ but is not inner invariant for $s$.

LEMMA 2.4. If a subspace $K$ is inner invariant for $T$ then $T(K \cap D)=K \cap T(D)$.

Proof. By the invariance of $K$ we get trivially that $T(K \cap D) \subseteq$ $K \cap T(D)$. To show $K \cap T(D) \subseteq T(K \cap D)$ let $y \in K \cap T(D)$ so that $y=T x$ for some $x$ in $D$. If $x \in D \backslash K$ and $T x \in K$ then we are contradicting (iii) in Definition 2.2.

THEOREM 2.5. If $T$ is one-to-one then the following are equivalent:

(i) $K$ is inner invariant for $T$;

(ii) $K$ is inner invariant for $T^{-1}$;

(iii) $K$ is invariant for both $T$ and $T^{-1}$.

Proof. We will just prove (i) $\Rightarrow$ (ii), the other cases being clear. Since $K$ is invariant, Lemma 2.4 implies that $T(K \cap D)=$ $K \cap T(D)$. Since $T$ is one-to-one, $T^{-1}$ exists and $D^{-1} \equiv T(D)$. Also $D=T^{-1}\left(D^{-1}\right)$.

Thus we have the following equalities:

$$
T(K \cap D)=K \cap T(D)=K \cap D^{-1}
$$

and $K \cap D=T^{-1}(K \cap T(D))=T^{-1}\left(K \cap D^{-1}\right)$ 
and

$$
K \cap T^{-1}\left(D^{-1}\right)=T^{-1}\left(K \cap D^{-1}\right) .
$$

Now, by the inner invariance of $K$ with respect to $T$ we have, by using (1) and (2), that

$$
\begin{aligned}
K & =\{T(K \cap D)+(K \cap D)\}^{-} \\
& =\left\{\left(K \cap D^{-1}\right)+T^{-1}\left(K \cap D^{-1}\right)\right\}^{-} .
\end{aligned}
$$

Hence condition (ii) in the definition of inner invariance (for $T^{-1}$ ) is satisfied.

We now use (3) and (4) to show $K$ is inner invariant for $T^{-1}$. First, from (4) we get (automatically)

$$
T^{-1}\left(K \cap D^{-1}\right) \subseteq K
$$

so that (i) of the definition of invariance is satisfied for $T^{-1}$.

Assume $x \in D^{-1}$ and $T^{-1} x \in K$. In order for condition (iii) in the definition of inner invariance to hold for $T^{-1}$ we must show $x \in K$. If $x$ did not belong to $K$, then this, with the assumptions that $x \in D^{-1}$ and $T^{-1} x \in K$, would be a contradiction to condition (iii) and therefore to inner invariance.

Now $T^{-1} x \in K$ and $T^{-1} x \in T^{-1}\left(D^{-1}\right)$ since $x \in D^{-1}$. Therefore $T^{-1} x \in K \cap T^{-1}\left(D^{-1}\right)=T^{-1}\left(K \cap D^{-1}\right)$ by (3). The operator $T^{-1}$ is oneto-one so that $x$ must belong to $K \cap D^{-1}$ and hence is in $K$. Thus $K$ is inner invariant for $T^{-1}$.

The next two examples exhibit (1) operators, all of whose invariant subspaces are inner invariant and (2) operators without inner invariant subspaces. The latter settles the inner invariant subspace problem easily in contradistinction with the long standing but recently solved problem concerning the existence of invariant subspaces of bounded operators. (At the August, 1976 meeting of the American Mathematical Society in Toronto, Per Enflo announced that he had solved the invariant subspace problem.)

EXAMPle 2.6. Consider the bounded Volterra operator $V$ on $L^{2}(0,1)$ defined by $V f(x)=\int_{0}^{x} f(y) d y$. Kalisch [10] proved that the proper invariant subspaces of this operator are of the form $L^{2}(a, 1)$ for $0<a<1$. We show that these subspaces are also inner invariant for $V$. Since $L^{2}(a, 1)$ is invariant, we need only demonstrate the last 
condition in the definition of inner invariance. Thus let $a$ be fixed and assume that $0 \neq f \in L^{2}(0,1)$ with $f \notin L^{2}(a, 1)$. We must show that $V f \notin L^{2}(a, 1)$ but this is obvious since $f$ must have some of its support on $(0, a)$ and therefore $V f(x)=\int_{0}^{x} f(y) d y$ must also have support on $(0, a)$. Thus $V f \notin L^{2}(a, 1)$.

EXAmPLE 2.7. Consider the Hardy space $H^{2}$ in the unit disk (i.e., $\{z \in \mathbf{C}|| z \mid<1\})$. On this space we will be concerned with the weighted shift operator $S$ defined by $S f(z)=z f(z / 2)$ for $f \in H^{2}$. Donoghue [3] showed that the proper invariant subspaces of $S$ are of the form $z^{n} H^{2}$ for $n=1,2,3, \cdots$ It is then trivial to show that none of these subspaces is inner invariant.

Definition 2.8. The closed subspace $K$ of $H$ is said to be reducing for $T$ if:

(i) $\quad D(T)=(D \cap K) \oplus\left(D \cap K^{\perp}\right)$;

(ii) $T(K \cap D) \subseteq K$ and $T\left(K^{\perp} \cap D\right) \subseteq K^{\perp}$;

(iii) $\{T(K \cap D)+(K \cap D)\}^{-}=K$ or $\left\{T\left(K^{\perp} \cap D\right)+\left(K^{\perp} \cap D\right)\right\}^{-}=$ $K^{\perp}$.

This definition is a natural extension of the definition of invariance (Definition 2.1) to the concept of reducing, but it is not the standard definition used for unbounded operators. In Akhiezer and Glazman [1], page 82, a closed subspace $K$ is said to reduce a linear operator $T$ if only conditions (i) and (ii) of Definition 2.8 hold. In this case we will say that $K$ is $A$-reducing for $T$.

Proposition 2.9. Let $\overline{D(T)}=H$. Then $K$ reduces $T$ iff it $A$ reduces $T$.

\section{Proof. Straightforward.}

Remark. In general, $A$-reducing (in the absence of the density assumption) does not imply reducing.

Proposition 2.10. Let $T$ be one-to-one with $\overline{D(T)}=H$. Then if $K$ reduces $T$, both $K$ and $K^{\perp}$ are inner invariant for $T$.

Proof. Since $\overline{D(T)}=H$ we get trivially that $\{K \cap D\}^{-}=K$ and $\left\{K^{\perp} \cap D\right\}^{-}=K^{\perp}$. Hence both statements in condition (iii) of the definition of reducing are true so that we can conclude that both $K$ and $K^{\perp}$ are invariant for $T$. To prove inner invariance, let $x \in D \backslash K$ so that $x=k+k^{\perp}$ with $k \in K \cap D$ and $k^{\perp} \in K^{\perp} \cap D$. Then $T x=T k+T k^{\perp}$ where $T k \in K$ and $T k^{\perp} \in K^{\perp}$ since $K$ reduces $T$. We know $x \notin K$ so 
that $k^{\perp} \neq 0$. If $T x \in K$ then we must have $T k^{\perp}=0$ which would imply $k^{\perp}=0$, a contradiction. Hence $T x \notin K$. A similar argument works for $K^{\perp}$.

REMARK. If $D(T)$ were not dense in $H$ then only one of the two conditions in (iii) of Definition 2.8 might hold, in which case only one of $K$ or $K^{\perp}$ would be inner invariant.

Proposition 2.11. Let $T$ be one-to-one with $\overline{D(T)}=H$. Then if $K$ reduces $T, K$ is inner invariant for $T$ and so is invariant for T. Furthermore, in general, these implications do not hold in the other direction.

Proof. Proposition 2.10 states that the first implication is true and the second one is a simple consequence of the definition of inner invariance. To see that the implications do not hold in the other direction, consider Examples 2.6 and 2.3. In Example 2.6 we saw that the subspaces $L^{2}(a, 1)$ for $0<a<1$ are inner invariant for the Volterra operator $V$, but they do not reduce it since $L^{2}(a, 1)^{\perp}=L^{2}(0, a)$ is not invariant for $V$. In Example 2.3, we exhibited an operator with an invariant subspace that was not inner invariant.

Example 2.12. We show that if $T$ is not one-to-one then Propositions 2.10 and 2.11 may actually be false. Let $T_{1}$ be a linear operator on a finite dimensional Hilbert space $H_{1}$ with nonzero kernel. Let $T_{2}$ be a nonsingular linear operator on a finite dimensional Hilbert space $H_{2}$. Form the Hilbert space $H=H_{1} \oplus H_{2}$ and the operator $T=$ $T_{1} \oplus T_{2}$. The operator $T$ is not one-to-one since $T_{1}$ is singular. The subspace $\mathrm{H}_{2}$ clearly reduces $T$ but is not inner invariant for $T$. To see this consider $x=h_{1}+h_{2}$ with $h_{1} \in H_{1}, h_{2} \in H_{2}$ and $0 \neq h_{1} \in$ kernel of $T_{1}$; then $x \notin H_{2}$ but $T x=T_{1} h_{1}+T_{2} h_{2}=T_{2} h_{2} \in H_{2}$ so that $H_{2}$ is invariant but not inner invariant for $T$.

EXAMPLE 2.13. We show several things here. First we exhibit an operator that has inner invariant and (non inner) invariant subspaces. Second, the inner invariant subspaces will be totally ordered. Third, the (non inner) invariant subspaces are examples of subspaces for which condition (ii) in the definition of invariance holds nontrivially. This example extends Example 2.6 and so we use the same notation.

Since the point spectrum of $V$ is empty, $V^{-1}$ exists. We write $L^{2}$ for $L^{2}(0,1)$. Since $D(V)=L^{2}$ we conclude that $D\left(V^{-1}\right)=\left\{f \in L^{2} \mid f\right.$ is absolutely continuous, $f^{\prime} \in L^{2}$, and $\left.f(0)=0\right\}$ and $V^{-1} f(x)=$ $d / d x f(x)$. The operator $V^{-1}$ is a closed unbounded operator (Stone [15], Theorem 10.7, Page 428). 
As was shown in Example 2.6, the inner invariant subspaces of $V$ and $V^{-1}$ are the subspaces $L^{2}(a, 1)$ for $0<a<1$ and they are totally ordered. These do not constitute all the invariant subspaces for $V^{-1}$. It is straightforward to show that the spaces $P_{n}$, where $P_{n}$ is the set of all polynomials of degree less than or equal to the positive integer $n$, are invariant. As a matter of fact $P_{n} \cap D\left(V^{-1}\right)$ which is the linear subspace generated by $\left\{x, x^{2}, \cdots, x^{n}\right\}$ is properly contained in $P_{n}$, and $V^{-1}\left(P_{n} \cap D\left(V^{-1}\right)\right)=P_{n-1}$ is also properly contained in $P_{n}$ but $\left\{\left(P_{n} \cap\right.\right.$ $\left.\left.D^{-1}\right)+V^{-1}\left(P_{n} \cap D^{-1}\right)\right\}=P_{n}$ so that condition (ii) in the definition of invariance is indeed satisfied nontrivially.

We close this section with some propositions giving us certain conditions under which some or all of the concepts of invariance, inner invariance, and reducing coincide. In the following propositions the linear operator $A$ is assumed to be a bounded operator defined everywhere on the Hilbert space $H$.

Proposition 2.14. If $A$ is self-adjoint and one-to-one then the following three conditions are equivalent:

(i) $K$ is inner invariant for $A$;

(ii) $K$ is reducing for $A$;

(iii) $K$ is invariant for $A$.

Proof. By Proposition 2.11 all we need do is show that $K$ being invariant for $A$ implies $K$ is reducing for $A$.

If $x \in K$ and $y \in K^{\perp}$ then $0=(A x, y)$ since $K$ is invariant. But $(A x, y)=(x, A y)$ since $A=A^{*}$. Thus $A y$ is perpendicular to $x$. Since $x$ is arbitrary in $K$ we conclude that $A y \in K^{\perp}$ so that $K^{\perp}$ is also invariant for $A$ and thus reduces $A$.

Proposition 2.15. Let $A$ be a unitary operator on $H$. Then $K$ is inner invariant for $A$ iff $K$ is reducing for $A$.

Proof. $(\Leftarrow)$. Trivial.

( $\Rightarrow$ ). Theorem 2.5 tells us that if $K$ is inner invariant for $A$ then it is invariant for both $A$ and $A^{-1}$ and conversely. Let $x \in K$ and $y \in K^{\perp}$. Then since $K$ is invariant for $A^{-1}$, and $A$ is unitary, we get the following:

$$
0=\left(A^{-1} x, y\right)=\left(A^{*} x, y\right)=(x, A y) .
$$

Thus $A y$ is perpendicular to $K$, i.e., $A y \in K^{\perp}$ so that $K^{\perp}$ is invariant for $A$. Since both $K$ and $K^{\perp}$ are invariant for $A$, the subspace $K$ reduces $A$. 
Proposition 2.16. If $A$ is bounded and $A-1$ is generalized nilpotent then $A$ and $A^{-1}$ have the same closed invariant subspaces. Thus the inner invariant subspaces of $A$ (and $A^{-1}$ ) coincide with the invariant subspaces of $A$.

Proof. Consider the infinite series $\sum_{n=0}^{\infty}(1-A)^{n}$; this series converges if $\sum_{n=0}^{\infty}\left\|(1-A)^{n}\right\|$ converges and this series does converge since $A-1$ is generalized nilpotent. Since $\sum_{n=0}^{\infty}(1-A)^{n}=(1-(1-A))^{-1}=$ $A^{-1}$ we conclude that $A^{-1}$ exists and is bounded.

Hence if $K$ is invariant for $A$ then it is invariant for $1-A$ and therefore for $(1-A)^{n}$ for all positive integers $n$. Thus $K$ is invariant for $A^{-1}=\Sigma(1-A)^{n}$.

In the other direction we have

$$
A=\left(A^{-1}\right)^{-1}=\left(1-\left(1-A^{-1}\right)\right)^{-1}=\sum_{n=0}^{\infty}\left(1-A^{-1}\right)^{n}
$$

This is a valid expression for $A$ since

$$
\left(1-A^{-1}\right)^{n}=\left[A^{-1}(A-1)\right]^{n}=A^{-n}(A-1)^{n}
$$

and thus the generalized nilpotency of $A-1$ insures the convergence of $\sum_{n=0}^{\infty}\left(1-A^{-1}\right)^{n}$.

Thus, if $K$ is invariant for $A^{-1}$ then $K$ is invariant for $\left(1-A^{-1}\right)^{n}$ for all $n$, so that $K$ is invariant for $A$.

3. The shift operator. In this section we describe the inner invariant subspace structure of the shift operator in several settings. In the first setting, the spaces considered are the Hardy spaces $H^{p}$ for $1 \leqq p \leqq \infty$. For background on Hardy spaces, the reader is referrred to Hoffman [9].

Briefly, the Hardy spaces are the Banach spaces of $p$-integrable analytic functions in the unit disk $\{z|| z \mid<1\}$, or equivalently, the subspace of $L^{p}$ of the unit circumference with no negative Fourier coefficients.

Definition 3.1. The shift operator $U$ on $H^{p}$ is defined by $U f(z)=$ $z f(z)$ for $f \in H^{p}$.

LEMMA 3.2. The function $z-a(a \in \mathbf{C})$ is an outer function for all a of modulus 1.

Proof. We show that $z-a$ is outer by showing that $\log \left|e^{i \theta}-a\right|$ is 
integrable and that

$$
0 \equiv \log |-a|=\frac{1}{2 \pi} \int_{-\pi}^{\pi} \log \left|e^{i \theta}-a\right| d \theta
$$

It is clear that $z-a$ belongs to $H^{1}$ since it is bounded and analytic in the unit disk. Thus $\log \left|e^{i \theta}-a\right|$ must be integrable.

Let $a=1$ and set $F(z)=z-1$ so that $\log |F(0)|=0$. With a little calculation we get

$$
\begin{aligned}
\frac{1}{2 \pi} \int_{-\pi}^{\pi} \log \left|F\left(e^{i \theta}\right)\right| d \theta & =\frac{1}{4 \pi} \int_{-\pi}^{\pi} \log [2(1-\cos \theta)] d \theta \\
& =\log 2+\frac{1}{2 \pi} \int_{0}^{2 \pi} \log \left(\sin \frac{\theta}{2}\right) d \theta \\
& =0=\log |\dot{F}(0)| .
\end{aligned}
$$

Thus $z-1$ is outer. The case $F(z)=z-a(|a|=1)$ may then be reduced to the case $a=1$ by a simple change of variable and thus yields the same result.

We now describe the inner invariant subspaces of translates of the shift, i.e., inner invariant subspaces of $U-a$ for $a \in \mathbf{C}$.

THEOREM 3.3. The nonzero closed inner invariant subspaces $S$ of $U-a$ on $H^{p}(1 \leqq p \leqq \infty)$ are the following:

(i) If $|a|<1$ then $S$ is inner invariant for $U-a$ iff $S=g H^{p}$ where $g$ is an inner function and $g(a) \neq 0$.

(ii) If $|a| \geqq 1$ then $S$ is inner invariant for $U-a$ iff $S=g H^{p}$ where $g$ is an inner function; i.e., $S$ is inner invariant iff $S$ is invariant.

Proof. Since Beurling [2] showed that the invariant subspaces of $U$ (and therefore of $U-a$ ) are of the form $g H^{p}$ for $g$ inner, all we need do is test which of these satisfy condition (iii) in the definition of inner invariance. We divide this into several cases.

Case 1. Let $|a|<1$ and assume $g(a) \neq 0$. Given $f$ in $H^{p} \backslash g H^{p}$ we must show that $(U-a) f \notin g H^{p}$ in order for $g H^{p}$ to be inner invariant for $U-a$. Equivalently, if $(U-a) f \in g H^{p}$ we must show that $f \in$ $g H^{p}$. Thus assume that there is a function $h \in H^{p}$ such that $(U-a) f(z)=g(z) h(z)$, i.e.,

$$
(z-a) f(z)=g(z) h(z) .
$$


Therefore

$$
f(z)=g(z) h(z) / z-a .
$$

Now the left hand side of (5) is zero when $z=a$ so that $g(a) h(a)=$ 0 , but $g(a) \neq 0$ by assumption, hence $h(a)=0$. In other words $h(z) / z-a$ is analytic. Then, by using (6), in order for $f$ to belong to $g H^{p}$ we need only show

$$
\lim _{r \rightarrow 1} \int_{-\pi}^{\pi}\left|\frac{h\left(r e^{i \theta}\right)}{r e^{i \theta}-a}\right|^{p} d \theta<\infty
$$

Now, the inequality

$$
\frac{1}{\left|r e^{i \theta}-a\right|^{p}} \leqq \frac{1}{|r-| a||^{p}}
$$

implies that

$$
\frac{1}{2 \pi} \int_{-\pi}^{\pi} \frac{\left|h\left(r e^{i \theta}\right)\right|^{p}}{\left|r e^{i \theta}-a\right|^{p}} d \theta \leqq \frac{1}{|r-| a||^{p}} \cdot \frac{1}{2 \pi} \int_{-\pi}^{\pi}\left|h\left(r e^{i \theta}\right)\right|^{p} d \theta
$$

and therefore

$$
\begin{aligned}
\lim _{r \rightarrow 1} \frac{1}{2 \pi} \int_{-\pi}^{\pi}\left|\frac{h\left(r e^{i \theta}\right)}{r e^{i \theta}-a}\right|^{p} d \theta & \leqq \frac{1}{(1-|a|)^{p}} \lim _{r \rightarrow 1} \frac{1}{2 \pi} \int_{-\pi}^{\pi}\left|h\left(r e^{i \theta}\right)\right|^{p} d \theta \\
& =\frac{1}{(1-|a|)^{p}}\|h\|_{p}^{p}<\infty
\end{aligned}
$$

since $h \in H^{p}$. From this we conclude that $h / z-a$ is in $H^{p}$ so that $f=g h$ is in $g H^{p}$. This means $g H^{p}$ is inner invariant for $U-a$ provided $g(a) \neq 0$.

Case 2. Let $|a|<1$ and assume $g(a)=0$. Since $g(a)=0$ and $g \in H^{p}$, the proof in Case 1 above (with $h$ replaced by $g$ ) shows that $g / z-a$ is in $H^{p}$. At the same time $g / z-a$ is not in $g H^{p}$ since $1 / z-a$ is not in $H^{p}$. Thus $g / z-a$ is in $H^{p} \backslash g H^{p}$ and $(U-a) g(z) / z-a=g(z)$ is in $g H^{p}$ which contradicts the definition of inner invariance. Therefore $g H^{p}$ cannot be inner invariant for $U-a$ when $g(a)=0$.

These first two cases prove part (i).

Case 3. Let $|a|>1$. In this case it is obvious that all invariant subspaces of $U-a$ are inner invariant. 
Case 4. Let $|a|=1$. We assume that $(z-a) f \in g H^{p}$ or

$$
(z-a) f(z)=g(z) h(z)
$$

for some $h$ in $H^{p}$. Since $f$ and $h$ are in $H^{p}$ which is contained in $H^{1}$, we know that there is a decomposition of $f$ and $h$ unique up to constants of modulus 1 such that

$$
f=g_{1} F_{1} \quad \text { and } \quad h=g_{2} F_{2}
$$

where $g_{1}$ and $g_{2}$ are inner functions and $F_{1}$ and $F_{2}$ are outer functions in $H^{p}$.

Substituting (8) into (7) we get

$$
(z-a) g_{1} F_{1}=g g_{2} F_{2} \text {. }
$$

Then since $z-a$ is outer (Lemma 3.2) and bounded we can conclude that $(z-a) F_{1}$ is outer. Since the decomposition of an $H^{p}$ function is unique up to constants of modulus 1 , we can conclude from (9) that $g_{1}=c g g_{2}$ and $(z-a) F_{1}=b F_{2}$ for some $c, b$ in $\mathbf{C}$ with $|c|=1=$ $|b|$. Therefore $F_{1}=b F_{2} / z-a \in H^{p}$ and this implies that $F_{2} / z-a \in$ $H^{p}$. Hence $h / z-a=g_{2} F_{2} / z-a \in H^{p}$ so that $f=g h / z-a$ is in $g H^{p}$. This means that $g H^{p}$ is inner invariant for $U-a$.

Proposition 3.4. (i) If $|a|<1$ then $\left\{(z-a) H^{2}\right\} \varsubsetneqq H^{2}$.

(ii) If $|a|=1$ then $\left\{(z-a) H^{2}\right\}^{-}=H^{2}$.

(iii) If $|a|>1$ then $(z-a) H^{2}=H^{2}$.

Proof. If $|a|>1$ then $1 / z-a$ is in $H^{2}$ so that $f=(z-a) f / z-a$ is in $(z-a) H^{2}$, i.e., $(z-a) H^{2}=H^{2}$. If $|a|=1$ then $z-a$ is an outer function (Lemma 3.2). We know that $(z-a) H^{2} \supseteq\left\{(z-a) z^{n}\right\}_{n=0}^{\infty}$ but it is also true that this sequence spans $H^{2}$ since $z-a$ is outer. Therefore $\left\{(z-a) H^{2}\right\}^{-}=H^{2}$. If $|a|<1$ then the function

$$
g(z)=-\frac{\bar{a}}{|a|} \cdot \frac{z-a}{1-\bar{a} z}
$$

is an inner function and

$$
-\frac{\bar{a}}{|a|} \frac{1}{1-\bar{a} z}
$$

is analytic in the unit disk. Therefore $(z-a) H^{2}=g(z) H^{2}$ and $g(z) H^{2}$ is a closed proper subspace of $H^{2}$. 
So far we have discussed the unilateral shift operator, i.e., the shift on $H^{p}$. Now we investigate the inner invariant subspace structure of the bilateral shift on $L^{2}(K)$ where $K$ is itself a Hilbert space.

We start by considering the special case where $K=\mathbf{C}$ so that $L^{2}(K)=L^{2}$ of the unit circumference, which we will abbreviate simply as $L^{2}$. Then the bilateral shift $U$ on either $L^{2}$ or $L^{2}(K)$ is defined by $U f(\theta)=e^{i \theta} f(\theta)$. We use $\chi_{A}$ to denote the characteristic function of a subset $A$ of the unit circumference.

First, we present the following lemma.

LeMma 3.5. Let $1 \leqq p \leqq \infty$, let $c>0$ and let $g \in H^{p}$. A necessary and sufficient condition that the function

$$
h(z)=\frac{g(z)}{(z-a)^{c}}
$$

(with $|a|=1$ ) be in $H^{p}$ is that $h\left(e^{i \theta}\right)$ belong to $L^{p}$ of the unit circumference.

Proof. This is a slight generalization of a well known result and we leave the straightforward calculation to the interested reader.

THEOREM 3.6. The closed nonzero inner invariant subspaces $S$ of $U-a(a \in C)$ on $L^{2}$ are of the following form:

(i) If $|a|<1$, then $S$ is inner invariant for $U-a$ iff $S=\chi_{A} L^{2}$ where $A$ is a Baire set of the unit circumference.

(ii) If $|a| \geqq 1$, then $S$ is inner invariant for $U-a$ iff $S=\chi_{A} L^{2}$ (for $A$ a Baire set) or $S=F H^{2}$ (for $F$ a measurable function on the unit circumference with modulus 1 ); i.e., iff $S$ is invariant for $U$.

Proof. It is well known that the invariant subspaces of $U$ are either of the form $\mathrm{FH}^{2}$ or $\chi_{\mathrm{A}} \mathrm{L}^{2}$. We now divide the proof into several cases.

Case 1. Let $|a|<1$. It is clear that invariant subspaces of the form $F H^{2}$ are not inner invariant for $U-a$ since $1 /\left(e^{i \theta}-a\right) \in L^{2}$ but not to $H^{2}$.

Now consider subspaces of the form $\chi_{A} L^{2}$ but these subspaces are always inner invariant since $1 /\left(e^{i \theta}-a\right)$ is bounded in $L^{2}$ (for $|a|>1$ too).

Case 2. Let $|a|>1$. Here $1 /\left(e^{i \theta}-a\right)$ belongs not only to $L^{2}$ but also to $H^{2}$ (since it is analytic). Thus all invariant subspaces of the form $F H^{2}$ (for $F$ with modulus 1 ) are inner invariant for $U-a$. The space $\chi_{A} L^{2}$ is inner invariant for $U-a$ when $|a|>1$, as was mentioned previously. 
Case 3. Let $|a|=1$. In this case $1 /\left(e^{i \theta}-a\right) \notin L^{2}$. We consider, for $f$ in $L^{2}$ and $g$ in $H^{2}$, the following:

$$
f(\theta)=F(\theta) g(\theta) /\left(e^{\imath \theta}-a\right) .
$$

Since $f \in L^{2}$ and $|F(\theta)|=1$, equation (10) implies that $g(\theta) /\left(e^{\imath \theta}-a\right) \in L^{2}$ with $g$ in $H^{2}$. Lemma 3.5 then tells us that $g(\theta) /\left(e^{i \theta}-a\right)$ is in $H^{2}$ so that $f \in F H^{2}$ and thus $F H^{2}$ is inner invariant.

Lastly, for some $f$ and $g$ in $L^{2}$ we consider $f(\theta)=$ $\chi_{A}(\theta) g(\theta) /\left(e^{i \theta}-a\right)=\chi_{A}(\theta)\left(\chi_{A}(\theta) g(\theta) / e^{i \theta}-a\right)=\chi_{A}(\theta) f(\theta)$ so that $f \in \chi_{A} L^{2}$ and thus $\chi_{A} L^{2}$ is inner invariant when $|a|=1$.

For background material on $L^{2}(K)$ the reader is referred to Fillmore [5], pages 31-44. We will use theorems from that work. Loosely speaking $L^{2}(K)$, for $K$ a Hilbert space, consists of all measurable functions $f$ from the unit circumference into $K$ such that

$$
\|f\|_{L^{2}(K)}=\frac{1}{2 \pi} \int_{0}^{2 \pi}\|f(\theta)\|_{K}^{2} d \theta<\infty .
$$

Our goal is to describe the inner invariant subspaces of $U-a$ on $L^{2}(K)$. We will write $M$ for a reducing subspace of $U$ on $L^{2}(K)$. It is known that $M$ has the form $M=\{f \mid f(\theta) \in M(\theta)$ a.e. $\}$ where $M(\theta)=$ $P(\theta) K$ and $P$ is the projection operator from $L^{2}(K)$ onto $M$. If $S$ is an invariant subspace of $U$, it is known that $S=M \oplus N$ where $M$ reduces $U$, the space $N$ is invariant for $U$ and $\bigcap_{k=0}^{\infty} U^{k}(N)=\{0\}$. More specifically, we can state that $N=V H^{2}(R)$ where $R$ is a closed subspace of $K$ and $V$ is a partial isometry on $L^{2}(K)$, with initial space $L^{2}(R)$, that commutes with $U$.

We now prove the following series of lemmas.

LEMMA 3.7. If $g$ is in $H^{2}(K)$ and $|a|=1$ then a necessary and sufficient condition for $h(\theta)=g(\theta) /\left(e^{i \theta}-a\right)$ to belong to $L^{2}(K)$ is that $h(\theta)$ be in $H^{2}(K)$.

Proof. This is clearly a further extension of Lemma 3.5. If $h$ is in $H^{2}(K)$ then it automatically belongs to $L^{2}(K)$; so assume $g$ is in $H^{2}(K)$ and $g /\left(e^{t \theta}-a\right)$ is in $L^{2}(K)$. Let $\left\{b_{n}\right\}_{n=0}^{\infty}$ be an orthonormal basis for $K$ and let $g_{n}(\theta)=\left(g(\theta), b_{n}\right)$; i.e., the inner product of $g$ with $b_{n}$. Then it is known that $g(\theta)=\sum_{n} g_{n}(\theta) b_{n}$ (convergence of this sum being in the norm of $K$ ) and that $g_{n}$ is in $H^{2}$. Since $\left\{b_{n}\right\}_{n=0}^{\infty}$ is an orthonormal basis for $K$ we get $\|g(\theta)\|_{K}^{2}=\sum_{n=0}^{\infty}\left|g_{n}(\theta)\right|^{2}$. From this we get (using Fatou's Lemma) 
that

$$
\begin{aligned}
\left\|g /\left(e^{i \theta}-a\right)\right\|_{L^{2}(K)}^{2} & =\frac{1}{2 \pi} \int_{0}^{2 \pi}\left\|g(\theta) /\left(e^{i \theta}-a\right)\right\|_{K}^{2} d \theta \\
& =\frac{1}{2 \pi} \int_{0}^{2 \pi} \sum_{n=0}^{\infty}\left|g_{n}(\theta) /\left(e^{i \theta}-a\right)\right|^{2} d \theta \\
& =\sum_{n=0}^{\infty} \frac{1}{2 \pi} \int_{0}^{2 \pi}\left|g_{n}(\theta) /\left(e^{i \theta}-a\right)\right|^{2} d \theta \\
& =\sum_{n=0}^{\infty}\left\|g_{n} /\left(e^{i \theta}-a\right)\right\|_{L^{2}}^{2}
\end{aligned}
$$

Since $g /\left(e^{i \theta}-a\right)$ is in $L^{2}(K)$ we can conclude that $g_{n} /\left(e^{i \theta}-a\right)$ is in $L^{2}$ for all $n$. Thus we now have $g_{n}$ in $H^{2}$ with $g_{n} /\left(e^{i \theta}-a\right)$ in $L^{2}($ for $|a|=1)$ and so we can conclude, by Lemma 3.5 , that $g_{n} /\left(e^{i \theta}-a\right)$ is in $H^{2}$ for all $n$. From this we get finally that $g /\left(e^{i \theta}-a\right)$ is in $H^{2}(K)$.

LEMMA 3.8. (i) If $|a|<1$, then $N$ is never inner invariant for $U-a$. (ii) If $|a| \geqq 1$, then $N$ is always inner invariant for $U-a$.

Proof. We are assuming $N=V H^{2}(R)$ with $V$ and $R$ as described above.

Case 1. Let $|a|<1$ and $x$ be in $R$. Then $x /\left(e^{i \theta}-a\right)$ is in $L^{2}(R) \backslash H^{2}(R)$. Now $V$ maps $L^{2}(R)$ into $L^{2}(K)$ so that $V\left(x / e^{i \theta}-a\right)$ is in $L^{2}(K)$. Thus

$$
\left(e^{i \theta}-a\right) V\left(x / e^{i \theta}-a\right)=V x
$$

since $V$ commutes with $U$ and hence with $U-a$. Since $x$ is in $R$ and $R$ is contained in $H^{2}(R)$ we get $V x$ in $H^{2}(R)$. This contradicts the inner invariance of $N$.

Case 2. Let $|a|>1$. Assume there is an $f$ in $L^{2}(K)$ such that

$$
\left(e^{i \theta}-a\right) f(\theta)=V(\theta) g(\theta)
$$

for some $g$ in $H^{2}(R)$. Since $|a|>1$, the function $1 /\left(e^{i \theta}-a\right)$ is analytic and bounded in the unit disk so that $g /\left(e^{i \theta}-a\right)$ is in $H^{2}(R)$. Thus (11) implies that

$$
\begin{aligned}
f(\theta) & =\frac{1}{e^{i \theta}-a} V(\theta) g(\theta)=\frac{1}{e^{i \theta}-a} V(\theta)\left[\left(e^{i \theta}-a\right) \frac{g(\theta)}{e^{i \theta}-a}\right] \\
& =V(\theta) \frac{g(\theta)}{e^{i \theta}-a}
\end{aligned}
$$


since $V$ commutes with $U-a$ on $L^{2}(R)$. We already know that $g(\theta) /\left(e^{\imath \theta}-a\right)$ is in $H^{2}(R)$ so that $f$ is in $V H^{2}(R)$. Thus $N$ is inner invariant for $U-a$ when $|a|>1$.

Case 3. Let $|a|=1$. Assume $f$ is in $L^{2}(K)$ and $g$ is in $H^{2}(R)$. We must show

$$
f(\theta)=\frac{1}{e^{\imath \theta}-a} V(\theta) g(\theta) \in V H^{2}(R)
$$

but

$$
\begin{aligned}
\|f\|_{L^{2}(K)}^{2} & =\frac{1}{2 \pi} \int_{0}^{2 \pi}\left\|\frac{1}{e^{i \theta}-a} V(\theta) g(\theta)\right\|_{K}^{2} d \theta \\
& =\frac{1}{2 \pi} \int_{0}^{2 \pi}\left|\frac{1}{e^{i \theta}-a}\right|^{2}\|V(\theta) g(\theta)\|_{K}^{2} d \theta \\
& =\frac{1}{2 \pi} \int_{0}^{2 \pi}\left|\frac{1}{e^{i \theta}-a}\right|^{2}\|g(\theta)\|_{R}^{2} d \theta
\end{aligned}
$$

since $V(\theta)$ is a partial isometry a.e. from $R$ into $K$.

Thus

$$
\begin{aligned}
\|f\|_{L^{2}(K)}^{2} & =\frac{1}{2 \pi} \int_{0}^{2 \pi}\left\|g(\theta) /\left(e^{i \theta}-a\right)\right\|_{R}^{2} d \theta \\
& =\left\|g /\left(e^{i \theta}-a\right)\right\|_{L^{2}(R)}^{2} .
\end{aligned}
$$

Since $f$ is in $L^{2}(K)$ this means $g /\left(e^{i \theta}-a\right)$ is in $L^{2}(R)$. Since $V$ commutes with $U-a$ on $L^{2}(K)$ we can rewrite (12) as

$$
f(\theta)=V(\theta) g(\theta) /\left(e^{i \theta}-a\right) .
$$

Since $g$ is in $H^{2}(R)$ and $g /\left(e^{i \theta}-a\right)$ is in $L^{2}(R)$, an application of Lemma 3.7 tells us that $g /\left(e^{i \theta}-a\right)$ is in $H^{2}(R)$ so that $f$ must be in $V H^{2}(R)$.

These lemmas now allow us to completely describe the inner invariant subspaces of the bilateral shift.

THEOREM 3.9. The closed nonzero inner invariant subspaces $S$ of $U-a$ on $L^{2}(K)$ are:

(i) If $|a|<1$ then $S$ is inner invariant for $U-a$ iff $S=M$ where $M$ is a reducing subspace for $U$, i.e., iff $S$ is reducing for $U-a$.

(ii) If $|a| \geqq 1$ then $S$ is inner invariant for $U-a$ iff $S=M \oplus N$ with $N=V H^{2}(R)$, i.e., iff $S$ is invariant for $U-a$. 
Proof. Lemma 3.8 (i) tells us $N$ is not inner invariant for $U-a$ when $|a|<1$, but $M$ being a reducing subspace for $U$ is clearly also a reducing subspace for $U-a$. Since $U-a$ is one-to-one, Proposition 2.10 tells us $M$ is inner invariant for $U-a$. The question is, can $M \oplus N$ be inner invariant in these circumstances?

Since $N$ is not inner invariant, there is an $x$ in $L^{2}(K) \backslash N$ such that $(U-a) x \in N$. We know $L^{2}(K)=M \oplus M^{\perp}$ with $N \subseteq M^{\perp}$. Thus we can write $x=m_{1}+m_{2}$ where $m_{1} \in M$ and $m_{2} \in M^{\perp}$, and $(U-a) x=$ $(U-a) m_{1}+(U-a) m_{2} \in N$. We know $(U-a) m_{1}$ is in $M$ since $M$ is reducing for $U-a$. Since $N$ is arthogonal to $M$ this means $(U-a) m_{1}=0$ which in turn means $m_{1}=0$ since $U-a$ is one-to-one. Thus $x=m_{2} \in M^{\perp} \backslash N$ so that $x \notin M \oplus N$ yet $(U-a) x \in M \oplus N$ so that $M \oplus N$ cannot be inner invariant.

If $|a| \geqq 1$ then Lemma 3.8 (ii) tells us $N$ is inner invariant. It is also clear that $M$ is inner invariant. We will now show that $M \oplus N$ must also be inner invariant.

Assume $x \in L^{2}(K)$ and

$$
(U-a) x \in M \oplus N .
$$

The element $x$ can be expressed as $x=m_{1}+m_{2}$ as before, so that $(U-a) x$ is again equal to $(U-a) m_{1}+(U-a) m_{2}$. Since $M$ reduces $U-a$, we know

$$
(U-a) m_{2} \in M^{\perp} \quad \text { and } \quad(U-a) m_{1} \in M,
$$

so that $(U-a) m_{2}=(U-a) x-(U-a) m_{1} \in M \oplus N$. Therefore $(U-a) m_{2} \in M^{\perp} \cap(M \oplus N)=N$. Since $N$ is inner invariant for $U-a$ we can conclude that $m_{2}$ is in $N$.

Let us look a bit more closely at part (ii) of the previous theorem. The subspace $S$ has the form $M \oplus N$. If $K$ were one dimensional, i.e., $K=\mathbf{C}$ then this theorem should reduce to Theorem 3.6 (ii). It is not difficult to show that in this case the subspace $M$ reduces to $\chi_{A} L^{2}$ (for $A$ a Baire set) and $N$ reduces to $F H^{2}$ (for $F$ a measurable function of modulus 1). At this point there seems to be an inconsistency. In Theorem 3.6 we have $S=F H^{2}$ or $S=\chi_{A} L^{2}$ (but not both together) while in Theorem 3.9 we seem to allow the possibility that $S=F H^{2} \oplus \chi_{A} L^{2}$. That there is no inconsistency follows from the following:

Assume $S=F H^{2} \oplus \chi_{A} L^{2}$. This means $F H^{2}$ is contained in the orthogonal complement of $\chi_{A} L^{2}$, i.e., if $B$ is the complement of $A$ on the unit circumference, then $F H^{2} \subseteq \chi_{B} L^{2}$. If $A$ has measure zero then there 
is no problem since, in this case, $\chi_{A} L^{2}=\{0\}$ and $S=F H^{2}$. Thus, let us assume that the measure of $A$ is positive. Let $f=F g \in F H^{2}$ with $g$ in $H^{2}$. Then since $F H^{2} \subseteq \chi_{B} L^{2}$ we must necessarily conclude that $f=0$ on $A$, i.e., $f=0$ on a set of positive measure. Since $F$ has modulus 1 a.e., it is necessary that $g=0$ on a set of positive measure. This is impossible if $g$ is not identically zero.

Since $g \in H^{2}, \quad g \in H^{1}$ and the $\log$ of an $H^{1}$ function is integrable. But $\log |g|=-\infty$ on a set of positive measure which contradicts the integrability of $\log |g|$. Hence $g$ must be identically zero. This implies $F H^{2}=\{0\}$ so that $S=\chi_{A} L^{2}$. Thus when $K=\mathbf{C}$ it cannot happen that $S=M \oplus N$ nontrivially.

Lastly, there is a question that still remains open, namely, what are the inner invariant subspaces of the unilateral shift and its translates on $H^{2}(K)$ ? The invariant subspaces we know: they are of the form $N=V H^{2}(R)$ where $V$ is a partial isometry on $H^{2}(K)$ commuting with the unilateral shift and $R$ is a closed subspace of $K$. The argument used in Lemma 3.8, Cases 2 and 3, with minor modification, suffices to tell us that all invariant subspaces of $U$ are inner invariant for $U-a$ when $|a| \geqq 1$, but what happens when $|a|<1$ ? Things are not too clear in this case. In the one-dimensional case $S=F H^{2}$ and $S$ is inner invariant for $U-a$ iff $F(a) \neq 0$. What condition on the partial isometry $V$ on $H^{2}(R)$ reduces to $V(a) \neq 0$ when $K$ is one-dimensional?

4. A characterization of the Volterra operator. We characterize integration abstractly on an arbitrary Hilbert space. Then we apply the work of the previous section to obtain the inner invariant subspace structure for a translate of the integration operation.

For the sake of convenience we will write $A \sim B$ to mean $A$ is unitarily equivalent to $B$.

Definition 4.1. The Volterra operator $V$ is defined by $V f(x)=$ $\int_{0}^{x} f(y) d y$ for $f_{0}$ in $D(V)$ where $D(V)=\left\{f \in L^{2}(0, \infty) \mid V f \in L^{2}(0, \infty)\right\}$.

A straightforward application of the Fubini Theorem tells us that

$$
(V-a)^{-1} f(x)=-\frac{1}{a} f(x)-\frac{1}{a^{2}} \int_{0}^{x} e^{(x-t) / a} f(t) d t
$$

for $a$ in $\mathbf{C}-\{0\}$ where $f$ is in $D(V-a)^{-1}$. It is then not difficult to show that $(V-a)^{-1}$ is bounded and defined everywhere in $L^{2}(0, \infty)$ for $\operatorname{Re}(a)<0$.

Proposition 4.2. The Volterra operator $V$ is a closed densely defined unbounded operator. 
Proof. Since $(V+1)^{-1}$ is bounded and defined everywhere, it is closed. Therefore $V+1$ and $V$ are also closed.

Sarason [13] showed that $(V+1)^{-1}$ on $L^{2}(0, \infty)$ was unitarily equivalent to $(1+U) / 2$ on $H^{2}$ where $U$ is the shift operator. Since $(V+1)^{-1} \sim$ $(U+1) / 2$ we get $V+1 \sim 2(U+1)^{-1}$. Therefore $V+1$ (and hence $V$ ) is densely defined if $(U+1)^{-1}$ is, but $D(U+1)^{-1}=(U+1) D(U)=$ $(z+1) H^{2}$ and Proposition 3.4 (ii) assures us that $(z+1) H^{2}$ is dense in $H^{2}$.

To show $V$ is unbounded, define $f_{a}(x)$ to be 1 if $0 \leqq x \leqq a$ and $-a(a+1) /(x+1)^{2}$ if $x>a$ where $1<a<\infty$. Then $\left\|V f_{a}\right\|>a\left\|f_{a}\right\|$ for $a=2,3,4, \cdots$ so that $V$ is indeed unbounded.

In order to characterize the Volterra operator it will be useful to generalize the concept of a symmetric operator and its Cayley transform.

Definition 4.3. A linear operator $A$ on a Hilbert space $H$ is called $b$-symmetric if $e^{i b} A \subset A^{*}$ where $b$ is real. If $b=\pi$ then $A$ is called skew-symmetric.

We now note that virtually all results about symmetric operators and their Cayley transforms also hold for $b$-symmetric operators. Simply substitute $b$-symmetric for symmetric in their proofs. A good reference for symmetric operators is Akhiezer and Glazman [1].

Recall that if $B$ is a symmetric operator then its Cayley transform $C$ is defined by $C=(B-z)(B-\bar{z})^{-1}$ where $\operatorname{Im}(z)>0$ and $C$ is a partial isometry.

We now define a Cayley transform for a $b$-symmetric operator. If $A$ is $b$-symmetric then $B=e^{i b / 2} A$ is symmetric and has a Cayley transform $C$ where (for $\operatorname{Im}(z)>0$ )

$$
\begin{aligned}
C & =(B-z)(B-\bar{z})^{-1} \\
& =\left(e^{i b / 2} A-z\right)\left(e^{i b / 2} A-\bar{z}\right)^{-1} \\
& =\left(A-e^{-i b / 2} z\right)\left(A-e^{-i b / 2} \bar{z}\right)^{-1} .
\end{aligned}
$$

Now let $w=e^{-i b / 2} z$ so that

$$
C=C(A) \equiv(A-w)\left(A-e^{-i b} \bar{w}\right)^{-1}
$$

for $\operatorname{Im}\left(e^{i b / 2} w\right)>0$.

Definition 4.4. We call the partial isometry $C=C(A)$, i.e., (13) above, the Cayley transform of the $b$-symmetric operator $A$. The domain $D(C)=\left(A-e^{-i b} \bar{w}\right) D(A)$ and the range $R(C)=(A-w) D(A)$ where $\operatorname{Im}\left(e^{i b / 2} w\right)>0$. 
Definition 4.5. If $A$ is a $b$-symmetric operator, we define the defect spaces $H_{w}^{+}$and $H_{w}^{-}$by

$$
\begin{aligned}
& H_{w}^{+}=D(C)^{\perp}=\left\{\left(A-e^{-i b} \bar{w}\right) D(A)\right\}^{\perp} ; \\
& H_{w}^{-}=R(C)^{\perp}=\{(A-W) D(A)\}^{\perp}
\end{aligned}
$$

where $\operatorname{Im}\left(e^{i b / 2} w\right)>0$.

Definition 4.6. The defect indices of a $b$-symmetric operator $A$ are $(p, q)$ where $p=$ dimension $H_{w}^{+}$and $q=\operatorname{dimension} H_{w}^{-}$. From Definition 4.6 it is clear that $(p, q)$ are also the defect indices of the Cayley transform $C(A)$.

Proposition 4.7. Let $A$ be $b$-symmetric and $\operatorname{Im}\left(e^{i b / 2} w\right)>$ 0 . Then $H_{w}^{+}=\left\{x \in H \mid A^{*} x=e^{i b} w x\right\}, H_{w}^{-}=\left\{x \in H \mid A^{*} x=\bar{w} x\right\}$ and $D(A) \cap H_{w}^{+}=D(A) \cap H_{w}^{-}=H_{w}^{+} \cap H_{w}^{-}=\{0\}$.

THEOREM 4.8. Let $A$ be b-symmetric, then $A$ is closed iff $D\left(A^{*}\right)=$ $D(A) \oplus H_{w}^{+} \oplus H_{w}^{-}$where $\operatorname{Im}\left(e^{i b / 2} w\right)>0$. The algebraic direct sum above is not necessarily orthogonal.

Proof. $(\Rightarrow)$ This direction is well known and its proof can be found in Akhiezer and Glazman [1], Volume II, Page 98.

$(\Leftarrow)$ This part is new and its proof is due to Robert Waterman. We assume that $A$ is not closed and that $C=C(A)$ is the Cayley transform of $A$. We will also make use of the following equivalent statements:

$A$ is not closed $\Leftrightarrow C(A)$ is not closed $\Leftrightarrow D(C)$ is not closed $\Leftrightarrow$ $R(C)$ is not closed.

Since $C$ is not closed we can get a smallest closed extension $\hat{C}$ of $C$ by considering the naturally induced partial isometry on $\overline{D(C)}$ that extends $C$. It is then clear that $(C-1) D(C)=D(A)$ and $D(A)$ is dense in $H$. Thus $(\hat{C}-1) D(\hat{C})$ is also dense in $H$ since $(\hat{C}-1) D(\hat{C})=$ $(\hat{C}-1) \overline{D(C)} \supset(C-1) D(C)$. In this case $\hat{A} \equiv\left(e^{-t b} \bar{w} \hat{C}-w\right)(\hat{C}-1)^{-1}$ is a closed $b$-symmetric operator. We know $\hat{A} \supsetneqq A$ since $\hat{C} \supsetneqq C$ (see [1], Volume II, page 96). Since $\hat{A}$ is a closed $b$-symmetric operator, the first half of this theorem tells us $D\left(\hat{A}^{*}\right)=D(\hat{A}) \oplus H_{w}^{+}(\hat{A}) \oplus H_{w}^{-}(\hat{A})$. From Definition 4.5 we get $H_{w}^{+}(\hat{A})=D(\hat{C})^{\perp}=\widehat{D(C)^{\perp}}=D(C)^{\perp}=H_{w}^{+}(A)$ and $H_{w}^{-}(\hat{A})=R(\hat{C})^{\perp}=R(C)^{\perp}=R(C)^{\perp}=H_{w}^{-}(A)$. Therefore

$$
D\left(\hat{A}^{*}\right)=D(\hat{A}) \oplus H_{w}^{+}(A) \oplus H_{w}^{-}(A) \supsetneqq D(A) \oplus H_{w}^{+}(A) \oplus H_{w}^{-}(A)
$$


since $A \varsubsetneqq \hat{A}$. But $A \varsubsetneqq \hat{A}$ implies $\hat{A}^{*} \varsubsetneqq A^{*}$ so that $D\left(\hat{A}^{*}\right) \varsubsetneqq D\left(A^{*}\right)$. This completes the proof since by (14) $D\left(A^{*}\right) \supsetneqq D\left(\hat{A}^{*}\right) \supsetneqq D(A) \oplus H_{w}^{+} \oplus H_{w}^{-}$.

Definition 4.9. A $b$-symmetric (or partial isometry) $A$ is simple if there does not exist a closed subspace $K$ of $H$ invariant under $A$ such that $A$ restricted to $K$ is $b$-adjoint (or unitary). An operator $F$ is $b$-adjoint if $e^{i b} F=F^{*}$.

Remark. A $b$-symmetric operator $A$ is simple iff $C(A)$ is simple.

Let $H$ be a separable Hilbert space and let $\left\{\boldsymbol{e}_{i}\right\}_{i=1}^{\infty}$ be an orthonormal basis for $H$. We define an operator $C^{*}$ by

$$
C^{\#} e_{k}=e_{k+1} \quad \text { for } \quad k=1,2,3, \cdots
$$

It is clear that $C^{\#}$ is a partial isometry with defect indices $(0,1)$. As a matter of fact $C^{*}$ is the shift operator. A short calculation then shows that $\left(C^{*}-1\right) D\left(C^{*}\right)$ is dense in $H$ so that

$$
A^{*}=\left(e^{-i b} \bar{w} C^{*}-w\right)\left(C^{*}-1\right)^{-1}
$$

for $\operatorname{Im}\left(e^{i b / 2} w\right)>0$ is a $b$-symmetric operator.

THEOREM 4.10. (J. von Neumann) If a simple b-symmetric operator $A$ on $H$ has defect indices $(0,1)$ then $H$ is separable and $A$ is unitarily equivalent to $A^{\#}$.

REMARK. This important theorem was originally proved for symmetric operators. Its present form is a direct generalization of this and its proof is similar.

THEOREM 4.11. The Volterra operator $V$ on $L^{2}(0, \infty)$ is a simple skew-symmetric operator with defect indices $(0,1)$.

Proof. In Sarason [13], the following was shown: If $U$ is the shift operator on $H^{2}$ then $(V+1)^{-1} \sim(U+1) / 2$. A little calculation then gives $U \sim(1-V)(1+V)^{-1}=-C(V)$. Therefore $V$ is simple with defect indices $(0,1)$ since $U$ is, and $V$ is skew-symmetric since it is the "anti"-Cayley transform of $U$ (i.e., $b=\pi$ and $w=1$ in (13) above).

As a corollary of the last two theorems we now have the abstract characterization of the Volterra operator.

Corollary 4.12. Any skew-symmetric operator A, defined on a 
Hilbert space $H$, that has defect indices $(0,1)$ is unitarily equivalent to the Volterra operator $V$ on $L^{2}(0, \infty)$.

Proof. By Theorems 4.11 and 4.10 we have $V \sim A^{*}$ and $A \sim A^{*}$ so that $V \sim A$.

Since $V f(x)=\int_{0}^{x} f(y) d y$ for $f$ in $D(V)=\left\{f \in L^{2} \mid V f \in L^{2}\right\}$, we easily get that $V^{-1} g(x)=d / d x g(x)$ for $g$ in $D\left(V^{-1}\right)=\left\{f\right.$ in $L^{2} \mid f^{\prime} \in L^{2}$, $f \in A$.C. and $f(0)=0\}$ where $f^{\prime}(x)=d / d x f(x)$ and $A$.C. is the set of absolutely continuous functions on $L^{2}=L^{2}(0, \infty)$.

THEOREM 4.13. The operator $V$ is unitarily equivalent to $V^{-1}$.

Proof. In the proof of Theorem 4.11 we showed that $C(V) \sim$ - $U$. Thus since $U \sim e^{i b} U$ for real $b$ we get that

$$
\begin{aligned}
V & =(-C(V)-1)(C(V)-1)^{-1} \\
& \sim(U-1)(-U-1)^{-1} \\
& =(1-U)(1+U)^{-1} \\
& \sim\left(1-e^{i b} U\right)\left(1+e^{i b} U\right)^{-1} \\
& =(1+U)(1-U)^{-1} \quad \text { if } \quad b=\pi \\
& =\left\{(1-U)(1+U)^{-1}\right\}^{-1} \\
& \sim\{V\}^{-1}=V^{-1} .
\end{aligned}
$$

We finish this section with some remarks about the nature of the adjoint $V^{*}$ of the Volterra operator $V$. On $L^{2}(0,1), V f=\int_{0}^{x} f(y) d y$ and $V^{*} f=\int_{x}^{1} f(y) d y$. At a first glance it might seem natural to suppose that $V^{*}$ on $L^{2}(0, \infty)$ would be similarly defined, that is, $V^{*} f=$ $\int_{x}^{\infty} f(y) d y$. This is not the case.

Since by Theorems 4.11 and $4.2, V$ is a closed skew-symmetric operator, we can use Theorem 4.8 to deduce that $D\left(V^{*}\right)=$ $D(V) \oplus H_{1}^{+} \oplus H_{1}^{-}$. The defect indices of $V$ are $(0,1)$ so that $H_{1}^{+}=$ $\{0\}$. We now need to describe the defect space $H_{1}^{-}$. The function $e^{-t}$ in $L^{2}(0, \infty)$ is not in $D(V)$ since $V\left(e^{-t}\right)=1-e^{-t}$ and the constant 1 does not belong to $L^{2}(0, \infty)$. On the other hand $e^{-t}$ does belong to $D\left(V^{*}\right)$ since

$$
\left(V f, e^{-t}\right)=\int_{0}^{\infty} V f(t) e^{-t} d t=\lim _{b \rightarrow \infty} \int_{0}^{b} f(t) e^{-t} d t=\left(f, e^{-t}\right)
$$


for $f$ in $D(V)$. Thus $e^{-t}$ is in $D\left(V^{*}\right)$ and $V^{*} e^{-t}=e^{-t}$ so that $e^{-t}$ is in $H_{1}^{-}$. Since $H_{1}^{-}$is one dimensional, we conclude $H_{1}^{-}=\left[e^{-t}\right]$.

Proposition 4.17. The adjoint $V^{*}$ can be described by $V^{*} f(x)=$ $\lim _{b \rightarrow \infty} \int_{x}^{b} f(y) d y$ for $f$ in $D\left(V^{*}\right)=D(V) \oplus\left[e^{-t}\right]$.

Proof. From above we know $V^{*} e^{-x}=e^{-x}=\lim _{b \rightarrow \infty} \int_{x}^{b} e^{-t} d t$. If $f$ is in $D(V)$ then (using integration by parts) we have $(V f, f)=$ $\lim _{b \rightarrow \infty}|V f(b)|^{2}-(f, V f)$ which implies that $\lim _{b \rightarrow \infty} V f(b)=0$ for otherwise $\|V f\|=\infty$ which contradicts $f$ being in $D(V)$. Hence

$$
0=\lim _{b \rightarrow \infty} \int_{0}^{b} f(t) d t=\int_{0}^{x} f(t) d t+\lim _{b \rightarrow \infty} \int_{x}^{b} f(t) d t
$$

so that

$$
\lim _{b \rightarrow \infty} \int_{x}^{b} f(t) d t=-V f(x)=V^{*} f(x)
$$

since $V$ is skew-symmetric.

We can actually describe $D\left(V^{*}\right)$ in slightly different terms, namely $D\left(V^{*}\right)=\left\{f \in L^{2}(0, \infty) \mid V^{*} f \in L^{2}(0, \infty)\right\}$. It is obvious that $D\left(V^{*}\right)$ is contained in this set, which we will call $D^{*}$. We need only show $D^{*} \subset D\left(V^{*}\right)$. To do this consider $f$ in $D(V)$ and $g$ in $D^{*}$. Then

$$
\begin{aligned}
(V f, g) & =\int_{0}^{\infty} V f(x) \overline{g(x)} d x=\lim _{a \rightarrow \infty} \int_{0}^{a} V f(x) \overline{g(x)} d x \\
& =\lim _{a \rightarrow \infty} \int_{0}^{a} f(x) \int_{x}^{a} \overline{g(y)} d y d x
\end{aligned}
$$

and

$$
\lim _{a \rightarrow \infty} \int_{0}^{a} f(x) \int_{x}^{a} \overline{g(y)} d y d x=\int_{0}^{\infty} f(x) \overline{W g(x)} d x
$$

where $W=V^{*}$ with $D(W)=D$, so that

$$
(V f, g)=(f, W g)
$$

for $f$ in $D(V)$ and $g$ in $D^{*}$. 


\section{Inner invariant subspaces for Volterra type} operators. We are now ready to apply the results of the past few sections to the unbounded Volterra operator.

THEOREM 5.1. There exists a surjective isometry (i.e., a unitary map) I from $H^{2}$ to $L^{2}(0, \infty)$ such that the closed nonzero inner invariant subspaces of $V+1$ are precisely $\left\{I\left(g H^{2}\right) \mid g\right.$ is an inner function $\}$. The same result holds for $V^{-1}+1$ in place of $V+1$.

Proof. Recall that $V$ is a skew-symmetric simple operator with defect indices $(0,1)$. Thus $(1-V)(1+V)^{-1}$ is the shift operator since this is the Cayley transform of $V$. Therefore there is a unitary map $I$ from $H^{2}$ onto $L^{2}(0, \infty)$ such that $I U=(1-V)(1+V)^{-1} I$ where $U$ is the shift operator on $H^{2}$. Thus $I U+I=2(1+V)^{-1} I$ so that $I_{2}^{1}(U+1)=$ $(1+V)^{-1} I$. Thus $K$ is an inner invariant subspace of $\frac{1}{2}(U+1)$ iff $I(K)$ is an inner invariant subspace of $(1+V)^{-1}$, and $\frac{1}{2}(U+1)$ has the same inner invariant subspaces as $U+1$. Theorem 3.3 then tells us that $K$ must equal $g H^{2}$ with $g$ an inner function. Thus $\left\{I\left(g H^{2}\right) \mid g\right.$ inner $\}$ is the set of inner invariant subspaces for $(V+1)^{-1}$, and by Theorem 2.5 for $V+1$ too.

To get the second part of this theorem, notice that by Theorem 4.13 we have $V \sim V^{-1}$ so that $V+1 \sim V^{-1}+1$.

REMARK. It should be pointed out that while the unitary maps from $H^{2}$ on to $L^{2}(0, \infty)$ may be different for $V+1$ and $V^{-1}+1$ it happens that it is also possible to pick a single unitary map $I$ in such a way that the spaces $I\left(g H^{2}\right)$ are inner invariant for both $V+1$ and $V^{-1}+1$ simultaneously. For an example of such a unitary map see Sarason [13].

If we now look back at Example 2.6 we will notice that the spaces $L^{2}(a, 1)$ for $0 \leqq a \leqq 1$ are inner invariant for the Volterra operator $V$ on $L^{2}(0,1)$. Since our Volterra operator $V$ on $L^{2}(0, \infty)$ is a natural extension of this it seems appropriate to inquire if the spaces $L^{2}(a, \infty)$ are inner invariant for $V$.

Proposition 5.2. The subspaces $L^{2}(a, \infty)$, for $0<a<\infty$, of $L^{2}(0, \infty)$ are inner invariant for $V+1$.

Proof. We will show that $L^{2}(a, \infty)$ is invariant for both $V+1$ and $(V+1)^{-1}$. Then an application of Theorem 2.5 shows that $L^{2}(a, \infty)$ is inner invariant.

Since $(V+1)^{-1}$ is bounded and defined everywhere, we need only show that $(V+1)^{-1}\left(L^{2}(a, \infty)\right) \subseteq L^{2}(a, \infty)$ but $(V+1)^{-1} f(x)=$ $f(x)-\int_{0}^{x} e^{t-x} f(t) d t$ for $f$ in $L^{2}(a, \infty)$. Since $\int_{0}^{x} e^{t-x} f(t) d t$ has its support 
in $[a, \infty)$ if $f$ does, we can conclude that $(V+1)^{-1} f$ is indeed in $L^{2}(a, \infty)$.

To show $L^{2}(a, \infty)$ is invariant for $V+1$, we have

$$
\begin{aligned}
D(V+1) \cap L^{2}(a, \infty) & =D(V) \cap L^{2}(a, \infty) \\
& =\left\{f \in L^{2}(0, \infty) \mid \int_{0}^{x} f(y) d y \in L^{2}(0, \infty)\right\} \cap L^{2}(a, \infty) \\
& =\left\{f \in L^{2}(a, \infty) \mid \int_{a}^{x} f(y) d y \in L^{2}(a, \infty)\right\}
\end{aligned}
$$

and it is clear that this is dense. in $L^{2}(a, \infty)$ iff $D(V)$ is dense in $L^{2}(0, \infty)$. Thus condition (ii) in the definition of invariance is satisfied.

As in the first part of the proof, $\int_{0}^{x} f(y) d y$ has its support in $[a, \infty)$ if $f$ does. Thus if $f$ is in $D(V) \cap L^{2}(a, \infty)$ then $(V+1) f$ must be in $L^{2}(a, \infty)$.

REMARK. From Theorem 5.1, there must be an inner function $g_{a}$ such that $I\left(g_{a} H^{2}\right)=L^{2}(a, \infty)$. Using the isometry $I$ given by Sarason [13] we see that $g_{a}(z)=\exp (a(z+1) /(z-1))$ is precisely the inner function we want.

Proposition 5.3. The inner invariant subspaces of $V+1$ are all inner invariant for $V$.

Proof. In Proposition 4.2 we showed that $(V+1)^{-1} \sim(1+U) / 2$ so that $V+1 \sim 2(1+U)^{-1}$ or $V \sim 2(1+U)^{-1}-1$. Thus $I\left(g H^{2}\right)$ is inner invariant for $V$ iff $g H^{2}$ is inner invariant for $2(1+U)^{-1}-1$. Since $g H^{2}$ is inner invariant for $(1+U) / 2$ it is inner invariant for $2(1+U)^{-1}$ and therefore necessarily invariant for $2(1+U)^{-1}$. We must now show that $g H^{2}$ is also invariant for $2(1+U)^{-1}-1$.

Clearly $D\left(2(1+U)^{-1}-1\right)=D(1+U)^{-1}=(z+1) H^{2}$. By Proposition 3.4 we know that $(z+1) H^{2}$ is dense in $H^{2}$. Also since $g H^{2}$ is inner invariant for $U+1$ we get $(z+1) H^{2} \cap g H^{2}=(z+1) g H^{2}$ (we leave the proof of this fact to the interested reader). Now condition (ii) in the definition of invariance tells us that we must show $\left\{\left((z+1) H^{2} \cap g H^{2}\right)+\right.$ $\left.\left[2(U+1)^{-1}-1\right]\left((z+1) H^{2} \cap g H^{2}\right)\right\}^{-}=g H^{2}$. Since $\quad(z+1) H^{2} \cap g H^{2}=$ $g(z+1) H^{2}$ we know that this subspace is dense in $g H^{2}$ because $(z+1) H^{2}$ is dense in $H^{2}$. Thus condition (ii) will be satisfied if we can show $\left[2(U+1)^{-1}-1\right]\left(g(z+1) H^{2}\right) \subseteq g H^{2}$ (this is condition (i) of the definition of invariance) but this is true iff $2(U+1)^{-1} g(z+1) H^{2} \subseteq g H^{2}$ which is clearly true. Thus $I\left(g H^{2}\right)$ is invariant for $V$.

To complete this proof we now show that $I\left(g H^{2}\right)$ is also invariant for $V^{-1}$ and then call on Theorem 2.5. We do this by showing $V^{-1}+1 \sim$ 
$2(U-1)^{-1}$ so that $V^{-1} \sim 2(U-1)^{-1}-1$. Then we use the argument given above to show that $g H^{2}$ is indeed invariant for $2(U-1)^{-1}-1$.

To show $V^{-1}+1 \sim 2(U-1)^{-1}$, consider the fact that $(1+V)^{-1}=$ $1-K$ where $K g(x)=\int_{0}^{x} e^{t-x} g(t) d t$. We then consider the differential equation $\left(i V^{-1}+i\right) f=g$, that is, $i f^{\prime}(x)+i f(x)=g(x)$. The solution of this equation is $f(x)=i K g(x)$. Thus $f(x)=\left(i V^{-1}+i\right)^{-1} g(x)=i K g(x)$ or $-K=\left(V^{-1}+1\right)^{-1}$. Since $1+V \sim 2(1+U)^{-1}$ we have $1-K=$ $(1+V)^{-1} \sim(U+1) / 2$ or $-K \sim(U-1) / 2$. Since $\left(V^{-1}+1\right)^{-1}=-K$ we conclude $\left(V^{-1}+1\right) \sim 2(U-1)^{-1}$.

We now examine one difference between the bounded Volterra operator on $L^{2}(0,1)$ and the unbounded Volterra operator on $L^{2}(0, \infty)$. In reference [10], Kalisch showed that $V$ on $L^{2}(0,1)$ is a unicellular operator. For the unbounded Volterra operator, it is not.

It is not difficult to show this. We need only show that the spaces $I\left(g H^{2}\right)$ for $g$ an inner function are not totally ordered. This is true iff the spaces $g H^{2}$ are themselves not totally ordered. Consider two subspaces $g_{1} H^{2}$ and $g_{2} H^{2}$ of $H^{2}$ with

$$
g_{1}(z)=\frac{\bar{a}}{|a|} \cdot \frac{a-z}{1-\bar{a} z} \text { and } g_{2}(z)=\frac{\bar{b}}{|b|} \cdot \frac{b-z}{1-\bar{b} z}
$$

where $a$ and $b$ are nonzero complex numbers such that $a \neq b$. It is then a routine calculation to show that neither $g_{1} H^{2} \subseteq g_{2} H^{2}$ nor $g_{2} H^{2} \subseteq$ $g_{1} H^{2}$. Thus the unbounded Volterra operator is not unicellular.

We do know all of the inner invariant subspaces for $V+1$ but do we know all of them for $V$ ? The answer at present is no, though we can (and will) exhibit a rather large set of inner invariant subspaces of $V$ that are not inner invariant for $V+1$.

EXAMPLE 5.4. Let $P_{n}$ be the set of functions of the form $p(t) e^{-t}$ where $p(t)$ is a polynomial of degree at most $n$ for $n$ a positive integer. Thus $P_{n}$ is an $n+1$ dimensional subspace of $L^{2}(0, \infty)$. We will show that $P_{n}$ is inner invariant for $V^{-1}$ and therefore also for $V$. Now $V^{-1}=d / d t \quad$ with $\quad D\left(V^{-1}\right)=\left\{f \in L^{2}(0, \infty) \mid f \in A . C ., \quad f^{\prime} \in L^{2}(0, \infty)\right.$ and $f(0)=0\}$.

Claim 1. $P_{n} \cap D\left(V^{-1}\right)=\left\{p(t) e^{-t} \mid p(0)=0\right.$ and $p$ is a polynomial of degree at most $n$ \}.

Proof of claim. Straightforward.

Claim 2. $V^{-1}\left(P_{n} \cap D\left(V^{-1}\right)\right) \subseteq P_{n}$. 
Proof of claim. We have $V^{-1} p(t) e^{-t}=\left(p^{\prime}(t)-p(t)\right) e^{-t}$ which is in $P_{n}$ whether or not $p(0)=0$.

Claim 3. $P_{n}=\left\{\left(P_{n} \cap D\left(V^{-1}\right)\right)+V^{-1}\left(P_{n} \cap D\left(V^{-1}\right)\right)\right\}$.

Proof of claim. Straightforward.

These three claims taken together tell us that $P_{n}$ is invariant for $V^{-1}$. We now show that $P_{n}$ is inner invariant.

If $V^{-1} f=f^{\prime}$ is in $P_{n}$ then $f^{\prime}(t)=p(t) e^{-t}$ with $p$ a polynomial of degree at most $n$. Therefore

$$
\begin{aligned}
f(t)= & \int_{0}^{t} p(y) e^{-y} d y \\
= & -\left(p(t)+p^{\prime}(t)+p^{\prime \prime}(t)+\cdots+p^{(n)}(t)\right) e^{-t} \\
& +\left(p(0)+p^{\prime}(0)+\cdots+p^{(n)}(0)\right) .
\end{aligned}
$$

Since $f$ is in $L^{2}(0, \infty)$ we conclude $\sum_{k=0}^{n} p^{(k)}(0)=0$; hence $f(t)=$ $-\sum_{k=0}^{n} p^{(k)}(t) e^{-t}$. Thus $f(t)=g(t) e^{-t}$ where $g(t)$ is a polynomial of degree at most $n$, and so $f$ belongs to $P_{n}$ and $P_{n}$ is inner invariant for $V^{-1}$ and also for $V$.

We will show that the subspaces $P_{n}$ (for $n=1,2, \cdots$ ) are invariant for $V+1$ and $V^{-1}+1$ but not inner invariant for either. Keep in mind that $D\left(V^{-1}+1\right)=D\left(V^{-1}\right)$ so that $D\left(V^{-1}+1\right), 7 P_{n}=\left\{p(t) e^{-t} \mid p(0)=0\right\}$, where $p$ is understood to be a polynomial of degree at most $n$.

Let $f$ be in $D\left(V^{-1}+1\right) \cap P_{n}$ so that $f(t)=p(t) e^{-t}$ with $p(0)=$ 0 . Then

$$
\begin{aligned}
\left(V^{-1}+1\right) f(t) & =\left(V^{-1}+1\right) p(t) e^{-t} \\
& =p^{\prime}(t) e^{-t} .
\end{aligned}
$$

Thus, because of $(15)$ we have $\left(V^{-1}+1\right)\left(D\left(V^{-1}\right) \cap P_{n}\right)=P_{n-1}$. Further

$$
\begin{gathered}
\left(V^{-1}+1\right)\left(D\left(V^{-1}\right) \cap P_{n}\right)+\left(D\left(V^{-1}\right) \cap P_{n}\right) \\
=P_{n-1}+\left\{p(t) e^{-t} \mid p(0)=0\right\}=P_{n}
\end{gathered}
$$

since $t^{n} e^{-t} \in\left\{p(t) e^{-t} \mid p(0)=0\right\}$ and $P_{n-1}+\left[t^{n} e^{-t}\right]=P_{n}$. Thus (15) and (16) imply that $P_{n}$ is invariant for $V^{-1}+1$. On the other hand $t^{n+1} e^{-t}$ is in $D\left(V^{-1}+1\right) \backslash P_{n}$ while $\left(V^{-1}+1\right)\left(t^{n+1} e^{-t}\right)=(n+1) t^{n} e^{-t}$ belongs to $P_{n}$ so that $P_{n}$ is not inner invariant for $V^{-1}+1$.

We will use $L^{2}$ for $L^{2}(0, \infty)$ below. Consider $D(V+1)=D(V)=$ $\left\{f \in L^{2} \mid V f \in L^{2}\right\}$. We look at $D(V) \cap P_{n}$. Let $f$ be in $P_{n}$. Thus 
$f(t)=p(t) e^{-t}$. If $f$ is to belong to $D(V)$ then $\int_{0}^{t} p(y) e^{-y} d y$ must belong to $L^{2}$; but $\int_{0}^{t} p(y) e^{-y} d y=-e^{-t}\left(\sum_{k=0}^{n} p^{(k)}(t)\right)+\sum_{k=0}^{n} p^{(k)}(0)$. If this is to belong to $L^{2}$ then $\sum_{k=0}^{n} p^{(k)}(0)=0$ in which case $(V+1)\left(p(t) e^{-t}\right)=$ $-e^{-t}\left(\sum_{k=0}^{n} p^{(k)}(t)\right)+p(t) e^{-t}=-e^{-t}\left(\sum_{k=1}^{n} p^{(k)}(t)\right)$. Thus $\quad(V+1)$. $\left(P_{n} \cap D(V)\right) \subseteq P_{n}$ and $P_{n} \cap D(V)=\left\{p(t) e^{-t} \mid \sum_{k=0}^{n} p^{(k)}(0)=0\right\}$.

Claim 4. For $k=1,2, \cdots, n,\left(t^{n}-k t^{k-1}\right) e^{-t} \in P_{n} \cap D(V)$.

Proof of Claim. A simple calculation gives us

$$
\begin{aligned}
\sum_{i=0}^{k} p^{(t)}(t) & =\sum_{i=0}^{k} \frac{d^{i}}{d t^{i}}\left(t^{i}-i t^{i-1}\right) \\
& =t^{k}
\end{aligned}
$$

Therefore $\sum_{i=0}^{k} p^{i}(0)=0^{k}=0$.

Claim 5. $P_{n-1} \subseteq(V+1)\left(P_{n} \cap D(V)\right)$.

Proof of Claim. If we do some more calculation we get

$$
(V+1)\left(t^{k}-k t^{k-1}\right) e^{-t}=-e^{-t}\left(k t^{k-1}\right)
$$

for $k=1,2, \cdots, n$. Therefore $(V+1)\left(P_{n} \cap D(V)\right)$ contains the space generated by $e^{-t}, t e^{-t}, t^{2} e^{-t}, \cdots, t^{n-1} e^{-t}$ which is $P_{n-1}$.

Now let $p(t)=t^{n}-n !$ then clearly $\sum_{k=0}^{n} p^{k}(0)=-n !+n !=0$ so that $\left(t^{n}-n !\right) e^{-t}$ belongs to $P_{n} \cap D(V)$. From Claim 5 we get $n ! e^{-t}$ in $(V+1)\left(P_{n} \cap D(V)\right)$. Thus $t^{n} e^{-t}=\left(t^{n}-n !\right) e^{-t}+n ! e^{-t}$ and this belongs to $\left(P_{n} \cap D(V)\right)+(V+1)\left(P_{n} \cap D(V)\right)$ so that $P_{n}=\left(P_{n} \cap D(V)\right)+$ $(V+1)\left(P_{n} \cap D(V)\right)$. This means $P_{n}$ is invariant for $V+1$. From Claim 4 we know that $\left(t^{n+1}-(n+1) t^{n}\right) e^{-t}$ belongs to $D(V) \backslash P_{n}$ but $(V+1)$. $\left(t^{n+1}-(n+1) t^{n}\right) e^{-t}=-(n+1) t^{n} e^{-t}$ by (17) above, which is in $P_{n}$. Thus $P_{n}$ is not inner invariant for $V+1$.

A few comments about the inner invariant subspaces $I\left(g H^{2}\right)$ are now in order. We have shown that these subspaces fill out the set of inner invariant subspaces for $V+1$. We can also show that these subspaces are inner invariant for $V-a$ where $a$ is in the resolvent set of $V$. Since -1 belongs to the resolvent set and we do know all of the inner invariant subspaces for $V-(.-1)=V+1$, is it possible that the spaces $I\left(g H^{2}\right)$ also fill out the inner invariant subspace structure of $V-a$ ? If not, what other inner invariant subspaces are there? 
Proposition 5.5. The operators $V-a$ and $V^{-1}-a$, for $a \in \mathrm{C}$, have no nontrivial reducing subspaces.

Proof. It is well known that the shift operator on $H^{2}$ has no nontrivial reducing subspaces (see Hoffman [9], Page 110). Also, for a $b$-symmetric operator $A$, a subspace $K$ reduces $A$ iff it reduces $C(A)$ (see Akhiezer and Glazman [1]). Since $C(V)=-U$ where $V$ is the Volterra operator and $U$ is the shift, we can conclude that $V$ has no nontrivial reducing subspaces. Since $V \sim V^{-1}$, we also know that $V^{-1}$ has no nontrivial reducing subspaces. We know

$$
D(V-a)=D(V) \text { and } \overline{D(V)}=L^{2}(0, \infty)
$$

Let us assume that $K$ is a reducing subspace for $V-a$ where $a$ is a nonzero complex number. Therefore

$$
D(V-a)=(D(V) \cap K) \oplus\left(D(V) \cap K^{\perp}\right)
$$

by Definition 2.8 and (18) above. We therefore conclude that $\{K \cap D(V)\}^{-}=K$ and $\left\{K^{\perp} \cap D(V)\right\}^{-}=K \perp$. In this case $(V-a)(K \cap D(V)) \subseteq K \quad$ iff $\quad V(K \cap D(V)) \subseteq K, \quad$ and $(V-a)\left(K^{\perp} \cap D(V)\right) \subseteq K^{\perp}$ iff $V\left(K^{\perp} \cap D(V)\right) \subseteq K^{\perp}$ so that $K$ must also reduce $V$. Thus $K=\{0\}$ or $K=L^{2}(0, \infty)$. Since $V^{-1} \sim V$ we get the same result for $V^{-1}-a$.

6. Some applications. As in Goldberg's book, "Unbounded Linear Operators", it is possible to define a natural induced linear operator on a quotient space. To do this consider a linear operator $T$ with domain $D$ contained in a Hilbert space $H$. If $K$ is a closed subspace of $H$ we can consider the quotient space $H / K$. The elements of $H / K$ are equivalence classes of the form $x+K$ for $x$ in $H$. We will denote this equivalence class by $[x]_{k}$, or simply $[x]$ when no ambiguity results.

Definition 6.1. We define the induced operator $\hat{T}: H / K \rightarrow H / K$ by $\hat{T}_{k}[x]_{k}=[T x]_{k}$ where $D\left(\hat{T}_{k}\right)=\left\{[x]_{k} \mid \exists x_{0} \in[x]_{k}\right.$ with $\left.x_{0} \in D(T)\right\}$. When no ambiguity results we will use $\hat{T}$ for $\hat{T}_{k}$.

Proposition 6.2. A closed invariant subspace $K$ of $T$ is inner invariant for $T$ iff $\hat{T}_{k}$ is $1-1$.

Proof.

( $\Rightarrow$ ) Assume $\hat{T}[x]=\hat{T}[y]$. From Definition 6.1 we know $T x=$ 
$T y+k$ for some $k$ in $K$. Since $k=T(x-y)$ we conclude $k \in T(D) \cap K$, but the inner invariance of $K$ then implies $k \in T(K \cap D)$ so that there is a $k_{1}$ in $K \cap D$ such that $T k_{1}=k$. This tells us $T\left(x-y-k_{1}\right)=0$ so that $n=x-y-k_{1}$ is in the null space of $T$. But for an inner invariant subspace $K$, we always have the null space contained in $K \cap D$. Therefore $n \in K \cap D \quad$ so that $x=y+\left(n+k_{1}\right)$ with $n+k_{1} \in K$. This means $[x]=[y]$.

\section{$(\Leftarrow)$ Clear.}

Let $G$ be a subspace of $H$ that contains $K$. We will let $\hat{G}$ denote the subspace $G / K$ of $H / K$. Let $q_{k}$ be the natural homomorphism from $H$ onto $H / K$. Then $\hat{G}=q_{k}(G)$. It is clear that $q_{k}: G \rightarrow \hat{G}$ is 1-1. Denote $D(\hat{T})$ by $\hat{D}$.

THEOREM 6.3. Let $K$ be a closed invariant subspace of $T$. Let $G$ be closed with $G \supset K$. Then $G$ is (inner) invariant for $T$ iff $q_{k}(G)=\hat{G}$ is (inner) invariant for $\hat{T}_{k}$.

\section{Proof.}

$(\Rightarrow)$ Suppose $[g] \in \hat{G} \cap \hat{D}=(G \cap D) / K$. We can assume $g \in G \cap D$. Since $G$ is invariant for $T$, we get $T g \in G$. Thus $\hat{T}[g]=$ $[T g]$ is in $\hat{G}$, that is, $\hat{T}(\hat{G} \cap \hat{D}) \subset \hat{G}$. We must now show that $\hat{G}=$ $\{(\hat{G} \cap \hat{D})+\hat{T}(\hat{G} \cap \hat{D})\}$ but through elementary calculations we get

$$
\begin{aligned}
\hat{G}=G / K & =\{(G \cap D)+T(G \cap D)\}^{-} / K \\
& =\{[(G \cap D)+T(G \cap D)] / K\}^{-} \\
& =\{(G \cap D) / K+T(G \cap D) / K\}^{-} \\
& =\{(G \cap D) / K+\hat{T}[(G \cap D) / K]\}^{-} \\
& =\{(\hat{G} \cap \hat{D})+\hat{T}(\hat{G} \cap \hat{D})\}^{-} .
\end{aligned}
$$

$(\Leftarrow)$ Since $q_{k}$ is $1-1$ and onto from the subspaces of $H$ containing $K$ to the subspaces of $H / K$, we know that given $\hat{G}$ a subspace of $H / K$, there must exist a subspace $G$ containing $K$ such that $q_{k}(G)=\hat{G}$. We will assume $\hat{G}$ is invariant for $\hat{T}$ and show that $G$ must then be invariant for $T$.

Let $x \in G \cap D$ so that $[x] \in \hat{G} \cap \hat{D}$. Thus $\hat{T}[x] \in \hat{G}$ since $\hat{G}$ is invariant for $\hat{T}$. Therefore there is a $y \in[T x]$ such that $y \in G$. This means $T x-y=k \in K$ or that $T x=y+k \in G+K \subset G$. Thus $T(G \cap D) \subset G$. 
Since $\hat{G}$ is invariant for $\hat{T}$ we know

$$
\begin{aligned}
G / K=\hat{G}= & \{(\hat{G} \cap \hat{D})+\hat{T}(\hat{G} \cap \hat{D})\}^{-} \\
& =\{(G \cap D) / K+T(G \cap D) / K\}^{-} \\
& =\{[(G \cap D)+T(G \cap D)] / K\}^{-} \\
& =\{(G \cap D)+T(G \cap D)\}^{-} / K
\end{aligned}
$$

Since $q_{k}$ is $1-1$ we can conclude that $G=\{(G \cap D)+T(G \cap D)\}^{-}$as soon as we know that $\{(G \cap D)+T(G \cap D)\}^{-} \supset K$. This is true since $K$ being invariant for $T$ implies

$$
K=\{(K \cap D)+T(K \cap D)\}^{-} \subset\{(G \cap D)+T(G \cap D)\}^{-} .
$$

For inner invariance, assume $G$ is not inner invariant for $T$. Then there is an $x$ in $D \backslash G$ with $T x$ in $G$. This says $[x] \in \hat{D} \backslash \hat{G}$ with $\hat{T}[x] \in \hat{G}$ which contradicts the inner invariance of $\hat{G}$.

In the other direction we assume $\hat{G}$ not inner invariant for $\hat{T}$. Thus there is an $[x]$ in $\hat{D} \backslash \hat{G}$ with $\hat{T}[x] \in \hat{G}$. This means there is a $y$ in $[x]$ with $y \in D \backslash G$. Now $T y \in[T y]=\hat{T}[y]=\hat{T}[x] \in \hat{G}$. Thus there is a $z \in[T y]$ with $z$ in $G$. Hence $T y-z=k$ for some $k$ in $K$ so that $T y=z+k \in G$. Since $y \in D \backslash G$ this contradicts the inner invariance of $G$.

REMARK. Since $q_{k}$ is $1-1$ and onto, this theorem established a 1-1 correspondence between the (inner) invariant subspaces of $T$ containing $K$ and the (inner) invariant subspaces of $\hat{T}_{k}$.

We state the following straightforward result.

Corollary 6.4. Let $K$ be inner invariant for $T$ and let $G$ be a subspace containing $K$. Then the following conditions are equivalent:

(i) $G$ is inner invariant for $T$

(ii) $\hat{G}$ is inner invariant for $\hat{T}_{k}$

(iii) $\hat{G}$ is inner invariant for $\hat{T}_{k}^{-1}$

(iv) $\hat{G}$ is invariant for both $\hat{T}_{k}$ and $\hat{T}_{k}^{-1}$.

\section{REFERENCES}

1. N. I. Akhiezer, and I. M. Glazman, Theory of Linear Operators in Hilbert Space, Vols. I and II, Frederick Unger Publishing Company, New York, N.Y., Vol. I (1966), Vol. II (1963). 
2. A. Beurling, On two problems concerning linear transformations in Hilbert space, Acta Mathematica, 81 (1949).

3. W. F. Donoghue, Jr., The lattice of invariant subspaces of a completely continuous quasi-nilpotent transformation, Pacific J. Math., 7, (1957).

4. N. Dunford, and J. T. Schwartz, Linear Operators, Part I, Interscience Publishers, Inc., New York, N.Y., 1967.

5. Peter A. Fillmore, Notes on Operator Theory, Van Nostrand Reinhold Company, New York, N.Y., 1970.

6. I. M. Glazman, and N. I. Akhiezer, see [1] above.

7. Henry Helson, Lectures on Invariant Subspaces, Academic Press, New York, N.Y., 1964.

8. E. Hewitt, and K. Stromberg, Real and Abstract Analysis, Springer-Verlag, New York, N.Y., 1965.

9. Kenneth Hoffman, Banach Spaces of Analytic Functions, Prentice-Hall, Inc., Englewood Cliffs, N.J., 1962.

10. G. K. Kalisch, On similarity, reducing manifolds and unitary equivalence of certain Volterra operators, Ann. of Math., 66, No. 3, 1957.

11. Direct proofs of spectral representation theorems, J. Math. Anal. Appl., 8, 1964, 351-363.

12. H. L. Royden, Real Analysis, The Macmillan Company, New York, N.Y., 1963.

13. Donald Sarason, A remark on the Volterra operator, J. Math. Anal. Appl., 12, 1965, 244-246.

14. J. T. Schwartz, and N. Dunford, see [4] above.

15. M. H. Stone, Linear Transformations in Hilbert Space, Amer. Math. Soc., Providence, R.I., 1966.

16. K. Stromberg, and E. Hewitt, see [8] above.

Received May 30, 1975. Supported in Part by N.S.F. Grants \# 13288 and 21334 and by A.F.O.S.R. Grant \# 70-1870.

Glassboro State College

GLASSBORo, NJ 08028 


\section{PACIFIC JOURNAL OF MATHEMATICS}

\section{EDITORS}

RichaRd ARENS (Managing Editor)

University of California

Los Angeles, CA 90024

\section{R. A. Beaumont}

University of Washington

Seattle, WA 98105

C. C. Moore

University of California

Berkeley, CA 94720
J. DugunduI

Department of Mathematics

University of Southern California

Los Angeles, CA 90007

R. Finn AND J. Milgram

Stanford University

Stanford, CA 94305

\section{ASSOCIATE EDITORS}
E. F. BECKENBACH
B. H. NeumanN
F. WOLF
K. YoshidA

\section{SUPPORTING INSTITUTIONS}

UNIVERSITY OF BRITISH COLUMBIA

CALIFORNIA INSTITUTE OF TECHNOLOGY

UNIVERSITY OF CALIFORNIA

MONTANA STATE UNIVERSITY

UNIVERSITY OF NEVADA

NEW MEXICO STATE UNIVERSITY

OREGON STATE UNIVERSITY

UNIVERSITY OF OREGON

OSAKA UNIVERSITY

\author{
UNIVERSITY OF SOUTHERN CALIFORNIA \\ STANFORD UNIVERSITY \\ UNIVERSITY OF HAWAII \\ UNIVERSITY OF TOKYO \\ UNIVERSITY OF UTAH \\ WASHINGTON STATE UNIVERSITY \\ UNIVERSITY OF WASHINGTON \\ AMERICAN MATHEMATICAL SOCIETY
}

The Supporting Institutions listed above contribute to the cost of publication of this Journal, but they are not owners or publishers and have no responsibility for its contents or policies.

Mathematical papers intended for publication in the Pacific Journal of Mathematics should be in typed form or offset-reproduced (not dittoed), double spaced with large margins. Underline Greek letters in red, German in green, and script in blue. The first paragraph or two must be capable of being used separately as a synopsis of the entire paper. Items of the bibliography should not be cited there unless absolutely necessary, in which case they must be identified by author and Journal, rather than by item number. Manuscripts, in duplicate, may be sent to any one of the four editors. Please classify according to the scheme of Math. Reviews, Index to Vol. 39. All other communications should be addressed to the managing editor, or Elaine Barth, University of California, Los Angeles, California, 90024.

100 reprints are provided free for each article, only if page charges have been substantially paid. Additional copies may be obtained at cost in multiples of 50 .

The Pacific Journal of Mathematics is issued monthly as of January 1966. Regular subscription rate: $\$ 72.00$ a year (6 Vols., 12 issues). Special rate: $\$ 36.00$ a year to individual members of supporting institutions.

Subscriptions, orders for back numbers, and changes of address should be sent to Pacific Journal of Mathematics, 103 Highland Boulevard, Berkeley, California, 94708.

PUBLISHED BY PACIFIC JOURNAL OF MATHEMATICS, A NON-PROFIT CORPORATION

Printed at Jerusalem Academic Press, POB 2390, Jerusalem, Israel. 


\section{Pacific Journal of Mathematics}

Vol. 68, No. 2

April, 1977

William Allen Adkins, Aldo Andreotti and John Vincent Leahy, An analogue of Oka's theorem for weakly normal complex spaces ........

Ann K. Boyle, M. G. Deshpande and Edmund H. Feller, On nonsingularly

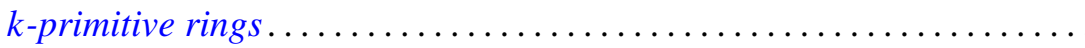

Rolando Basim Chuaqui, Measures invariant under a group of

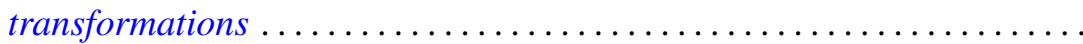

Wendell Dan Curtis and Forrest Miller, Gauge groups and classification of bundles with simple structural group .......................

Garret J. Etgen and Willie Taylor, The essential uniqueness of bounded nonoscillatory solutions of certain even order differential equations

Paul Ezust, On a representation theory for ideal systems

Richard Carl Gilbert, The deficiency index of a third order operator ........

John Norman Ginsburg, $S$-spaces in countably compact spaces using Ostaszewski's method.

Basil Gordon and S. P. Mohanty, On a theorem of Delaunay and some

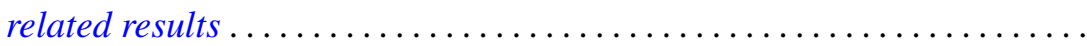

Douglas Lloyd Grant, Topological groups which satisfy an open mapping

theorem.

Charles Lemuel Hagopian, A characterization of solenoids

Kyong Taik Hahn, On completeness of the Bergman metric and its

subordinate metrics. II .

G. Hochschild and David Wheeler Wigner, Abstractly split group extensions.

Gary S. Itzkowitz, Inner invariant subspaces ...............

Jiang Luh and Mohan S. Putcha, A commutativity theorem for

non-associative algebras over a principal ideal domain.

Donald J. Newman and A. R. Reddy, Addendum to: "Rational approximation of $e^{-x}$ on the positive real axis".....

Akio Osada, On the distribution of a-points of a strongly annular function ....

Jeffrey Lynn Spielman, A characterization of the Gaussian distribution in a Hilbert space. .

Robert Moffatt Stephenson Jr., Symmetrizable-closed spaces ...

Peter George Trotter and Takayuki Tamura, Completely semisimple inverse $\Delta$-semigroups admitting principal series . . . . . . . .

Charles Irvin Vinsonhaler and William Jennings Wickless, Torsion free abelian groups quasi-projective over their endomorphism rings...

Frank Arvey Wattenberg, Topologies on the set of closed subsets ... 